\title{
Volume of supervised exercise training impacts glycaemic control in patients with type 2 diabetes: a systematic review with meta-regression analysis
}

\author{
D. Umpierre • P. A. B. Ribeiro • B. D. Schaan • J. P. Ribeiro
}

Received: 3 May 2012 / Accepted: 29 August 2012 /Published online: 16 November 2012

(C) Springer-Verlag Berlin Heidelberg 2012

\begin{abstract}
Aims/hypothesis Supervised exercise programmes improve glycaemic control in type 2 diabetes, but training characteristics associated with reduction in $\mathrm{HbA}_{1 \mathrm{c}}$ remain unclear. We conducted a systematic review with meta-regression analysis of randomised clinical trials (RCTs) assessing the association between intensity and volume of exercise training (aerobic, resistance or combined) and $\mathrm{HbA}_{1 \mathrm{c}}$ changes in patients with type 2 diabetes.

Methods Five electronic databases were searched (19802012) to retrieve RCTs of at least 12 weeks' duration, consisting of supervised exercise training vs no intervention, that reported $\mathrm{HbA}_{1 \mathrm{c}}$ changes and exercise characteristics. Two independent reviewers conducted study selection and data extraction.
\end{abstract}

Professor J. P. Ribeiro, who supervised this research, died on 23 August 2012, before publication of this study.

Electronic supplementary material The online version of this article (doi:10.1007/s00125-012-2774-z) contains peer-reviewed but unedited supplementary material, which is available to authorised users.

D. Umpierre $(\bowtie) \cdot$ P. A. B. Ribeiro · J. P. Ribeiro

Exercise Pathophysiology Research Laboratory,

Hospital de Clínicas de Porto Alegre,

Rua Ramiro Barcelos 2350,

Centro de Pesquisa Clínica-LaFiex, $3^{\circ}$ andar,

90035-003 Porto Alegre, RS, Brazil

e-mail: daniel.umpierre@gmail.com

B. D. Schaan

Endocrinology Division, Hospital de Clínicas de Porto Alegre,

Porto Alegre, Brazil

J. P. Ribeiro

Cardiology Division, Hospital de Clínicas de Porto Alegre,

Porto Alegre, Brazil

B. D. Schaan · J. P. Ribeiro

Department of Internal Medicine, Faculty of Medicine,

Universidade Federal do Rio Grande do Sul,

Porto Alegre, Brazil
Results Twenty-six RCTs (2,253 patients) met the inclusion criteria. In multivariate analysis, baseline $\mathrm{HbA}_{1 \mathrm{c}}$ and exercise frequency explained nearly $58 \%$ of between-study variance. Baseline $\mathrm{HbA}_{1 \mathrm{c}}$ was inversely correlated with $\mathrm{HbA}_{1 \mathrm{c}}$ reductions after the three types of exercise training. In aerobic training, exercise volume (represented by frequency of sessions) was associated with changes in $\mathrm{HbA}_{1 \mathrm{c}}$ (weighted $r=-0.64$ ), while no variables were correlated with glycaemic control induced by resistance training. In combined training, weekly volume of resistance exercise explained heterogeneity in multivariate analysis and was associated with changes in $\mathrm{HbA}_{1 \mathrm{c}}$ levels (weighted $r=-0.70$ ).

Conclusions/interpretation Reduction in $\mathrm{HbA}_{1 \mathrm{c}}$ is associated with exercise frequency in supervised aerobic training, and with weekly volume of resistance exercise in supervised combined training. Therefore, exercise volume is a major determinant of glycaemic control in patients with type 2 diabetes.

Keywords Aerobic exercise - Glycosylated haemoglobin A . Resistance training $\cdot$ Type 2 diabetes mellitus $\cdot$ Systematic review

$\begin{array}{ll}\text { Abbreviations } \\ 1-\mathrm{RM} & \text { One repetition maximum } \\ \mathrm{HR}_{\max } & \text { Maximum heart rate } \\ \text { PRISMA } & \begin{array}{l}\text { Preferred reporting items for systematic reviews } \\ \text { and meta-analyses }\end{array} \\ \mathrm{RCT} & \text { Randomised clinical trial } \\ \dot{V} \mathrm{O}_{2 \text { peak }} & \text { Peak oxygen uptake } \\ \mathrm{WMD} & \text { Weighted mean difference }\end{array}$

\section{Introduction}

Different exercise interventions benefit glycaemic control in patients with type 2 diabetes [1,2]. Systematic reviews have 
consistently shown that supervised exercise training is associated with absolute reduction in $\mathrm{HbA}_{1 \mathrm{c}}$ of approximately $0.6 \%$ [2-5]. Supervised aerobic, resistance or combined aerobic/resistance training are associated with greater decreases in $\mathrm{HbA}_{1 \mathrm{c}}$ than less strict strategies, such as exercise counselling alone [2, 6, 7]. Although important contributions have been made to our understanding of the impact of different types of exercise training for patients with type 2 diabetes [8, 9], investigations of the chronic impact on glycaemic control of the key characteristics of volume and intensity in structured exercise prescriptions are inconclusive.

As the manipulation of exercise training variables may optimise chronic glucose-lowering effects in different populations $[10,11]$, it would be useful to know which characteristics (e.g. frequency, duration of each session, total weekly duration and intensity) could be associated with greater benefits in patients with type 2 diabetes. A recent subanalysis from the multicentre Italian Diabetes Exercise Study showed that weekly exercise volume was positively related with improvements in quality of life in patients with type 2 diabetes [12], which would probably facilitate longterm adherence [13]. Supporting the importance of exercise volume, we have found that structured exercise durations of more than $150 \mathrm{~min} /$ week are associated with greater reductions in $\mathrm{HbA}_{1 \mathrm{c}}$ in type 2 diabetes [2], although previous data from aerobic training studies have indicated that exercise intensity, but not volume, is associated with reduction in $\mathrm{HbA}_{1 \mathrm{c}}$ levels [3]. Nonetheless, a 6 month trial has indicated that obese patients with type 2 diabetes have similar lowering of $\mathrm{HbA}_{1 \mathrm{c}}$ after aerobic training of either low-tomoderate or moderate-to-high intensity [14]. Furthermore, exercise volume and intensity in resistance training or combined aerobic/resistance programmes have not been studied so far.

Accordingly, this study consists of a systematic review with meta-regression analysis of randomised clinical trials (RCTs) on the associations of characteristics of supervised exercise training with changes in $\mathrm{HbA}_{1 \mathrm{c}}$ levels in patients with type 2 diabetes. Supervised exercise training is categorised according to whether it consists of aerobic exercise, resistance training or a combination of both.

\section{Methods}

Search strategy and eligibility criteria We conducted a structured literature search for studies published from January 1980 to June 2012. Electronic databases including MEDLINE (accessed by PubMed), Cochrane Central Register of Controlled Trials, EMBASE, SPORTdiscus and LILACS were searched. We also examined review articles and related references to identify other eligible studies.
Initially, searches included terms such as 'exercise', 'diabetes mellitus' and 'physical activity'. The advanced search strategy used in the PubMed database is published elsewhere [2]. Only eligible studies published in English, Portuguese or Spanish were included (electronic supplementary material [ESM] Fig. 1). A total of eight studies were excluded by language. This systematic review and metaanalysis is reported in accordance with the preferred reporting items for systematic reviews and meta-analyses (PRISMA) statement [15].

Included RCTs were required to have at least one arm of supervised exercise training (aerobic, resistance and/or a combination of both) in participants aged over 18 and with type 2 diabetes, compared with a control group. Studies were required to have evaluated $\mathrm{HbA}_{1 \mathrm{c}}$ as an outcome and reported means or differences between means and statistical dispersion values of $\mathrm{HbA}_{1 \mathrm{c}}$. As the present review was designed to address characteristics of exercise training, we only included trials with structured and supervised exercise programmes that were more likely to have had strict control of the variables of interest, such as intensity, session duration and frequency. We excluded studies that had nonhuman data, a follow-up period shorter than 12 weeks or included patients with type 1 diabetes or gestational diabetes; duplicate publications or substudies of included trials were also excluded.

Two reviewers (D. Umpierre and P. A. B. Ribeiro) independently assessed trials for inclusion in the systematic review. Disagreements were resolved by consensus or by a third reviewer (B. D. Schaan). Corresponding authors of potentially eligible studies were contacted if there was a suggestion that duplicated data were reported or data were missing.

The quality of the methods used in the included studies was evaluated by the independent reviewers, according to the PRISMA recommendation [16]. Assessment included adequate sequence generation, allocation concealment, blinding of outcomes assessors, use of intention-to-treat analysis and description of losses and exclusions. Studies without clear descriptions of an adequate sequence generation or how the allocation list was concealed were considered not to have fulfilled these criteria.

Data extraction In order to generate weighted mean differences (WMDs), absolute changes in $\mathrm{HbA}_{1 \mathrm{c}}$ as well as standard deviations were extracted from all studies. Whenever standard deviations or standard errors of the mean were not available, dispersion values were entered in the analysis by imputation methods.

Pertinent data regarding the details of the study methods, population characteristics, exercise training prescriptions and outcomes were extracted in standardised forms by the independent reviewers. The frequency of exercise (sessions/ 
week) and total time spent in exercise throughout the study, which are variables related to the training volume, were abstracted identically for the three types of supervised exercise training. In studies of aerobic training interventions, we also extracted the session duration, weekly exercise volume and relative exercise intensity (based on percentages of maximum heart rate $\left[\mathrm{HR}_{\max }\right]$ ) of each study. We used established equations to derive aerobic training intensities expressed as percentages of the peak oxygen uptake $\left(\dot{V} \mathrm{O}_{2 \text { peak }}\right)$ [17]. In resistance training interventions, we extracted the number of sets and repetitions, therefore calculating their weekly correspondence with the exercise frequency in each study. Resistance exercise intensities were computed as percentages of the one-repetition maximum (1-RM). In studies that used a range of repetitions to elicit maximal efforts at each set, the percentage values of the submaximal weight loads were obtained through an appropriate equation for the conversion from repetitions-to-fatigue to 1-RM [18]. In combined training studies, the characteristics of the aerobic and resistance components were extracted separately as described above for aerobic or resistance exercise training alone.

Statistical analysis Absolute changes in $\mathrm{HbA}_{1 \mathrm{c}}$ were treated as differences between arithmetic means before and after interventions. Thereafter, pooled WMDs were estimated by a random-effect model with the DerSimonianLaird method, and heterogeneity was assessed by Cochran $\mathrm{Q}$ and the $I^{2}$ statistic. Three comparisons were made, with each supervised exercise training group being compared with a group receiving no intervention, as follows: (1) aerobic exercise training vs control; (2) resistance exercise training vs control; and (3) combined exercise training vs control. As the present systematic review was designed to analyse the characteristics of supervised exercise programmes, we used only data from participants completing the follow-up.

In order to explore which exercise characteristics of volume and intensity would be potentially associated with larger changes in $\mathrm{HbA}_{1 \mathrm{c}}$ levels, we initially ran metaregressions by using the WMD estimates of $\mathrm{HbA}_{1 \mathrm{c}}$ and exercise training variables such as frequency of sessions, level of intensity, minutes of aerobic exercise per week and sets of resistance exercise per week. Significant clinical and/ or exercise variables in univariate models were also combined into multivariate meta-regression analyses. For each meta-regression model, the adjusted $r^{2}$ indicated the proportion of between-study variance explained by the covariates $[19,20]$. In addition, we generated correlations to test the association between $\mathrm{HbA}_{1 \mathrm{c}}$ WMDs with variables explaining heterogeneity in meta-regression analyses as well as with key exercise variables, based on clinical judgement of their importance. All correlation analyses were weighted by the inverse of the variance of each observation, and scatter 'bubble' plots were constructed to graphically display the proportional weights of the different trials.

In trials in which multiple exercise interventions were compared with a single control group, we split this shared control group into two or more groups, with smaller sample sizes weighted in relation to each exercise group. Therefore, comparisons were reasonably independent and overcame a unit-ofanalysis error for studies that could contribute to multiple and correlated comparisons [21]. All analyses were conducted using Stata 11.0 software (Stata, College Station, TX, USA).

\section{Results}

Systematic review and qualitative assessment After the exclusion of duplicate references, the search strategy resulted in 3,788 studies. Of these, 3,454 studies were excluded based on the titles and abstracts. Therefore, from 334 eligible articles that underwent full-text evaluation, 26 RCTs met the inclusion criteria and provided data on 2,253 patients. Of these, 935 patients were included in supervised aerobic exercise training, 249 in supervised resistance exercise training and 1,069 in supervised combined aerobic/resistance exercise training. The flow diagram of the search and major reasons for exclusions is shown in ESM Fig. 1.

ESM Table 1 summarises the characteristics of all the studies included [6, 8, 9, 22-44]. Trials were published from 1989 to June 2012, and the majority were small studies with sample sizes ranging from ten participants in the smallest to 563 in the largest study. The mean duration of interventions was 25 weeks (minimum-maximum: 12 to 52 ). The 2,253 patients had a mean age of 58 years (minimum-maximum: 53 to 69 ), mean baseline $\mathrm{HbA}_{1 \mathrm{c}}$ of $7.8 \%$ (minimum-maximum: $6.3 \%$ to $12.5 \%$ ) and mean diabetes duration of 6 years (minimummaximum: 2 to 10 years). In the quality assessment, $35 \%$ presented adequate sequence generation (nine of 26), 19\% reported allocation concealment (five of 26), 23\% had blinded assessment of outcomes (six of 26), 100\% described losses to follow-up and exclusions (26 of 26), and 19\% reported using the intention-to-treat principle for statistical analyses (five of 26) (ESM Table 2).

As shown in ESM Table 1, across 20 interventions of aerobic exercise training, [8, 9, 22-38] the mean exercise frequency was three sessions per week, mean session duration was $48 \mathrm{~min}$ (not including warm-up and cool down), and mean exercise intensity was $74 \%$ of the $\mathrm{HR}_{\max }$. In six trials $(33 \%)$, exercise intensity was transformed from submaximal rates of $\dot{V} \mathrm{O}_{2 \text { peak }}$ to the corresponding percentages of $\mathrm{HR}_{\max }$. All of the five trials using resistance exercise training $[8,9,26,39,40]$ had exercise frequency of three sessions per week. In these studies, the number of total sets 
per session ranged from 14 to 27 , and repetitions in each individual set ranged from eight to 20 . Regarding the weight loads used in each set, resistance exercise intensities ranged from $60 \%$ to $85 \%$ of the $1-\mathrm{RM}$. Across the ten trials of combined exercise training [6, 8, 9, 23, 26, 32, 41-44], the mean exercise frequency was three sessions per week (minimum-maximum: two to four sessions), mean session duration was 59 min (minimum-maximum: 45-90 min), mean aerobic exercise intensity was $73 \%$ of the $\mathrm{HR}_{\max }$ (minimum-maximum: $60-85 \%$ ), whereas resistance exercise intensity in each set ranged from $40 \%$ to $85 \%$ of the 1 -RM. We were unable to extract or derive exercise intensity in eight interventions of aerobic training [22-29] and three interventions of combined training [23, 26, 41]. These studies were included in the systematic review, but their data were not entered in the analyses assessing exercise intensity as a covariate.

Separate meta-analyses of studies with aerobic, resistance and/or combined exercise training were initially performed in order to generate effect sizes for each type of intervention. As expected, supervised exercise training was associated with improved glycaemic control, resulting in WMDs of $-0.70 \%(95 \%$ CI $-1.02,-0.38),-0.62$ $(95 \% \mathrm{CI}-1.14,-0.11)$ and $-0.47(95 \% \mathrm{CI}-0.64,-0.31)$ for aerobic, resistance and combined exercise training, respectively.

Meta-regression analyses Data from all trials indicated that baseline $\mathrm{HbA}_{1 \mathrm{c}}$, exercise frequency and weekly exercise volume partly explained the heterogeneity in the studies (Table 1). In the multivariate analysis, baseline $\mathrm{HbA}_{1 \mathrm{c}}$ and weekly exercise volume explained nearly $58 \%$ of the variance between studies. As shown in Fig. 1, higher baseline levels of $\mathrm{HbA}_{1 \mathrm{c}}$ were associated with greater $\mathrm{HbA}_{1 \mathrm{c}}$ reductions after the three types of exercise training (weighted $r=-0.52, p=0.001$ ).

In supervised aerobic training, univariate analysis demonstrated that exercise frequency explained nearly $32 \%$ of the between-studies variance, which was also confirmed by

Table 1 Univariate and multivariate meta-regression models

\begin{tabular}{|c|c|c|c|c|}
\hline Covariates & Coefficient & $95 \% \mathrm{CI}$ & $p$ & Adjusted $R^{2}$ \\
\hline \multicolumn{5}{|l|}{ Overall analysis: aerobic, resistance and combined training studies ${ }^{\mathrm{a}}$} \\
\hline Baseline $\mathrm{HbA}_{1 \mathrm{c}}(\%), n=26$ & -0.30 & $-0.48,-0.11$ & 0.003 & $44.5 \%$ \\
\hline Duration of diabetes (years), $n=19$ & -0.074 & $-0.16,0.12$ & 0.09 & NS \\
\hline Frequency (sessions/week), $n=26$ & -0.30 & $-0.53,-0.06$ & 0.02 & $31.6 \%$ \\
\hline Total time spent in exercise throughout the study (h), $n=26$ & -0.0009 & $-0.005,0.002$ & 0.6 & NS \\
\hline Weekly volume (min/week), $n=20$ & -0.004 & $-0.007,-0.00007$ & 0.04 & $15.5 \%$ \\
\hline Multivariate model: baseline $\mathrm{HbA}_{1 \mathrm{c}}+$ exercise frequency, $n=26$ & & & 0.001 & $57.6 \%$ \\
\hline \multicolumn{5}{|l|}{ Supervised aerobic training ${ }^{\mathrm{b}}$} \\
\hline Intensity $\left(\% \mathrm{HR}_{\max }\right), n=13$ & -0.003 & $-0.07,0.058$ & 0.9 & NS \\
\hline Frequency (sessions/week), $n=20$ & -0.69 & $-1.03,-0.35$ & $<0.001$ & $62.3 \%$ \\
\hline Weekly volume ( $\mathrm{min} /$ week), $n=20$ & -0.007 & $-0.014,0.0005$ & 0.07 & NS \\
\hline Multivariate model: baseline $\mathrm{HbA}_{1 \mathrm{c}}+$ exercise frequency, $n=20$ & & & 0.001 & $65.4 \%$ \\
\hline \multicolumn{5}{|l|}{ Supervised resistance training ${ }^{\mathrm{c}}$} \\
\hline Intensity $(\% 1-\mathrm{RM}), n=5$ & 0.13 & $-0.15,0.18$ & 0.8 & NS \\
\hline Weekly volume (sets/week), $n=5$ & 0.007 & $-0.05,0.06$ & 0.72 & NS \\
\hline Weekly volume (repetitions/week), $n=5$ & 0.0008 & -0.009 to 0.112 & 0.80 & NS \\
\hline \multicolumn{5}{|l|}{ Supervised combined training $\mathrm{d}$} \\
\hline Aerobic intensity $\left(\% \mathrm{HR}_{\max }\right), n=7$ & 0.04 & $-0.003,0.82$ & 0.07 & NS \\
\hline Aerobic volume ( $\mathrm{min} /$ week), $n=10$ & -0.0005 & $-0.01,0.12$ & 0.93 & NS \\
\hline Resistance intensity (\% 1-RM), $n=10$ & 0.007 & $-0.02,0.04$ & 0.62 & NS \\
\hline Resistance volume (sets/week), $n=10$ & -0.02 & $-0.033,0.001$ & 0.06 & NS \\
\hline Multivariate model: baseline $\mathrm{HbA}_{1 \mathrm{c}}+$ resistance volume, $n=10$ & & & 0.04 & $100 \%$ \\
\hline
\end{tabular}

Frequency, total time spent in exercise throughout the study, and weekly volumes of each type of training were considered characteristics of volume (amount). Percentages of either $\mathrm{HR}_{\max }$ or 1-RM were used to express intensity in aerobic and resistance exercise, respectively

$n$, number of studies assessed for each of the meta-regression analyses

${ }^{a}$ Overall unadjusted $I^{2}=79.8 \%, p$ for heterogeneity $<0.001$

${ }^{\mathrm{b}}$ Unadjusted $I^{2}=92.6 \%, p$ for heterogeneity $<0.001$

${ }^{\mathrm{c}}$ Unadjusted $I^{2}=88.7 \%, p$ for heterogeneity $<0.001$

${ }^{\mathrm{d}}$ Unadjusted $I^{2}=33.8 \%, p$ for heterogeneity $<0.14$ 


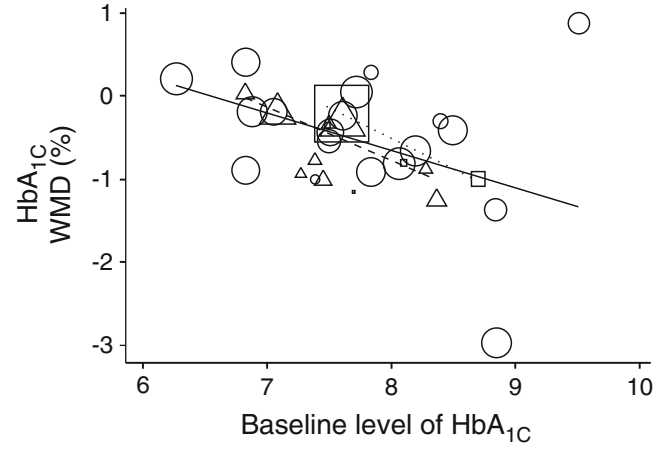

Fig. 1 Association between baseline levels of $\mathrm{HbA}_{1 \mathrm{c}}$ and changes in $\mathrm{HbA}_{1 \mathrm{c}}$ after different types of supervised exercise training. Each observation represents the WMD of $\mathrm{HbA}_{1 \mathrm{c}}$ between the different interventions of exercise training and control groups. The size of the symbols is proportional to the inverse variance of each study in the pooled analysis. Meta-regression lines are specific for each type of intervention: continuous line, aerobic training studies; dotted line, resistance training studies; and dashed line, combined training studies. Circles, supervised aerobic training; squares, supervised resistance training; triangles, supervised combined training. Slope for weighted regression, $y=-0.17 x+0.60$. Weighted correlation, $r=-0.52, p=0.001$. Note that $\mathrm{HbA}_{1 \mathrm{c}}$ levels are shown in \%. To convert values for $\mathrm{HbA}_{1 \mathrm{c}}$ in $\%$ into $\mathrm{mmol} / \mathrm{mol}$, subtract 2.15 and multiply by 10.929

a multivariate model including baseline $\mathrm{HbA}_{1 \mathrm{c}}$ and exercise frequency (Table 1). As depicted in Fig. 2a, aerobic exercise frequency was associated with changes in $\mathrm{HbA}_{1 \mathrm{c}}$, and showed a weighted regression slope of -0.39 , indicating that, for each additional aerobic exercise session per week, there was a corresponding decline of $0.39 \%$ in $\mathrm{HbA}_{1 \mathrm{c}}$ level (weighted $r=-0.64, p<0.01$ ). Interestingly, aerobic exercise intensity did not explain between-studies variance in the univariate analysis and showed no significant association with $\mathrm{HbA}_{1 \mathrm{c}}$ changes $(p=0.3$, Fig. $2 \mathrm{~b})$.

In trials of supervised resistance training, univariate meta-regression did not show any variables that would explain the heterogeneity between studies (Table 1), therefore limiting us to enter volume or intensity variables in the multivariate analysis. Likewise, there were no correlations between weekly resistance exercise volume or resistance exercise intensity with changes in $\mathrm{HbA}_{1 \mathrm{c}}$ levels (Fig. 3a, b).

In trials of supervised combined training, volume and intensity were analysed in relation to aerobic and resistance components separately. As shown in Table 1 and Fig. 4, the intensity of aerobic as well as of resistance exercises was not associated with changes in $\mathrm{HbA}_{1 \mathrm{c}}$. Likewise, metaregression and correlation analyses did not indicate association between aerobic exercise volume and $\mathrm{HbA}_{1 \mathrm{c}}$ changes induced by combined training. However, the weekly volume of resistance exercise in this type of training trended to significance in univariate analysis, and explained the heterogeneity between studies when subsequently combined with baseline $\mathrm{HbA}_{1 \mathrm{c}}$ in the multivariate meta-regression (Table 1). Figure $4 \mathrm{c}$ shows that resistance exercise volume
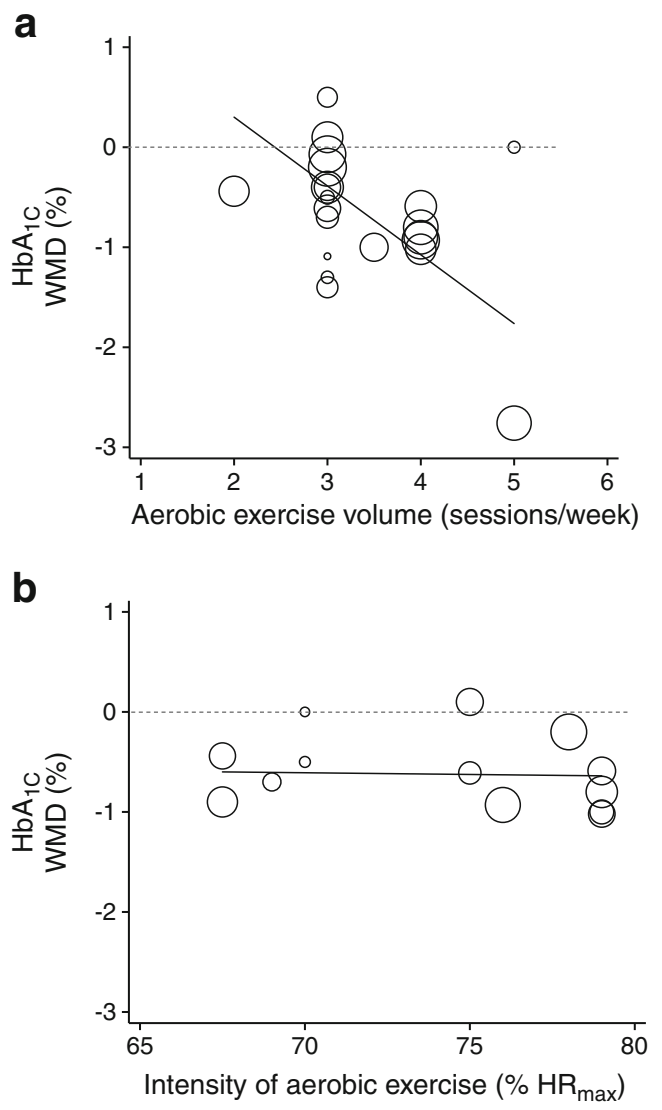

Fig. 2 Association between exercise volume (a) and intensity (b) with $\mathrm{HbA}_{1 \mathrm{c}}$ changes in studies of supervised aerobic training. Exercise volume is expressed as the frequency of sessions within a week and intensity is expressed as submaximal percentages of the $\mathrm{HR}_{\max }$. Each observation represents the WMD of $\mathrm{HbA}_{1 \mathrm{c}}$ between the aerobic training and control groups. The size of the circles is proportional to the inverse variance of each study in the pooled analysis. Slopes for weighted regressions, $y=-0.39 x+0.65$ and $y=-0.03+2.03$, for aerobic volume and intensity, respectively. Weighted correlations, $r=-0.64$, $p=0.002$ and $r=-0.32, p=0.3$ for aerobic volume and intensity, respectively

was inversely correlated with WMD in $\mathrm{HbA}_{1 \mathrm{c}}$ levels (weighted $r=-0.70, p=0.04$ ), showing a weighted regression slope of -0.02 , indicating that, for each additional resistance exercise set per week, there was a corresponding decrease of $0.02 \%$ in $\mathrm{HbA}_{1 \mathrm{c}}$ level after combined training interventions.

\section{Discussion}

The present systematic review provides an updated synthesis of the factors associated with improvements in glycaemic control induced by different types of supervised exercise training in type 2 diabetes. Overall, higher baseline $\mathrm{HbA}_{1 \mathrm{c}}$ levels were associated with greater reductions in $\mathrm{HbA}_{1 \mathrm{c}}$ levels after exercise interventions, supporting previous 
a

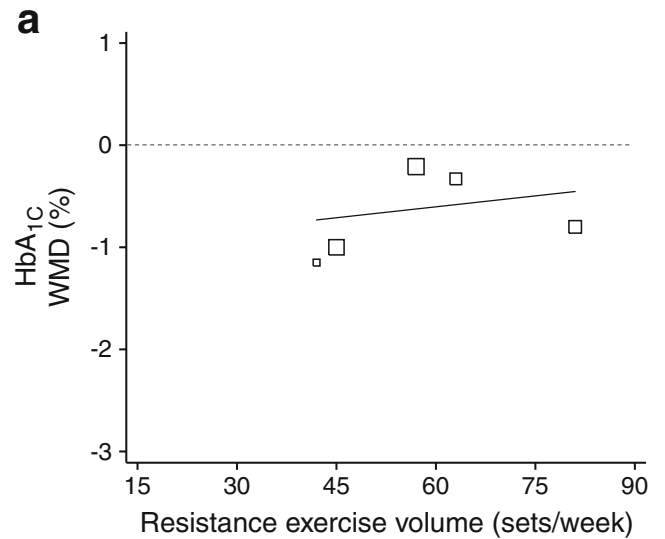

b

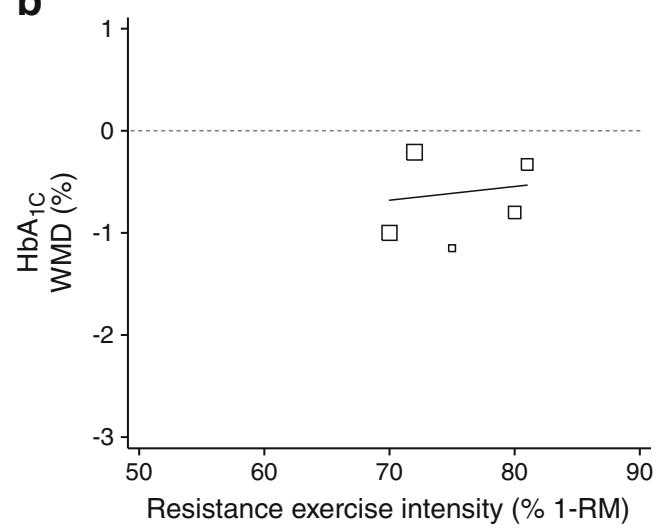

Fig. 3 Association between exercise volume (a) and intensity (b) with $\mathrm{HbA}_{1 \mathrm{c}}$ changes in studies of supervised resistance training. Exercise volume is expressed as the number of sets within a week and intensity is expressed as submaximal percentages of the 1-RM. Each observation represents the WMD of $\mathrm{HbA}_{1 \mathrm{c}}$ between the resistance training and control groups. The size of the squares is proportional to the inverse variance of each study in the pooled analysis. Slopes for weighted regressions, $y=-0.01 x-1.30$ and $y=-0.02-2.12$ for resistance volume and intensity, respectively. Weighted correlations, $r=0.26, p=0.7$ and $r=0.18, p=0.8$ for resistance volume and intensity, respectively

observations $[2,8,9]$. Although no exercise variables were found to be possible candidates to explain the effects of resistance training, the volume of exercise was associated with $\mathrm{HbA}_{1 \mathrm{c}}$ reductions in supervised aerobic training as well as in combined aerobic/resistance training studies. More specifically, we found that each aerobic exercise session added within a week may produce an additional reduction of $0.39 \%$ in $\mathrm{HbA}_{1 \mathrm{c}}$ level. Interestingly, as we explored the aerobic and resistance components of combined training studies separately, we found that volume of resistance exercise was the characteristic associated with change in $\mathrm{HbA}_{1 \mathrm{c}}$ level in this training modality.

In a sensitivity analysis published recently, we found that durations of weekly exercise longer than $150 \mathrm{~min}$ were associated with absolute reductions in $\mathrm{HbA}_{1 \mathrm{c}}$ of $0.89 \%$ in patients with type 2 diabetes [2]. Although this estimate was derived from a pooled analysis of studies with aerobic, resistance and combined exercise training, it highlighted an important role of the exercise volume in the exerciseinduced glycaemic improvements. In this context, the present study demonstrates that the frequency of exercise is the specific factor more likely to underlie the beneficial effects of aerobic training, meaning that the repetition of exercise sessions may be more important than longer or more intense sessions.

To our knowledge, only one previous systematic review with meta-analysis was designed to address quantitatively the characteristics of exercise training in type 2 diabetes [3]. In contrast with our findings on supervised aerobic training, that previous study [3] showed that weekly volume was not associated with glycaemic control, whereas exercise intensity was inversely correlated with $\mathrm{HbA}_{1 \mathrm{c}}$ levels after aerobic training interventions. We would attribute such conflicting results to fundamental differences in most of the studies included, which reflect distinct eligibility criteria regarding the minimal duration of exercise training interventions, training group assignments (as in the aerobic arm of the analysis, we entered groups performing aerobic exercise alone) and the number of studies analysed (an inherent limitation when comparing systematic reviews over time). Interestingly, a study has shown that 6 months of continuous aerobic training at low-to-moderate intensity or interval training at moderate-to-high intensities, matched for energy cost, induced similar $\mathrm{HbA}_{1 \mathrm{c}}$ reductions in patients with type 2 diabetes [14]. While an RCT [11] has indicated the importance of exercise volume for improvements in insulin sensitivity in non-diabetic individuals, other studies [45-47] have yielded controversial results on the role of intensity in aerobic programmes. In accordance with the findings of the present analysis, we have recently shown that lower- (55 \pm $2 \%$ of $\left.\mathrm{HR}_{\max }\right)$ and higher-intensity $\left(79 \pm 3 \%\right.$ of $\left.\mathrm{HR}_{\max }\right)$ aerobic training resulted in similar reductions in $\mathrm{HbA}_{1 \mathrm{c}}$ in patients with type 2 diabetes or metabolic syndrome [48]. However, aerobic training at a higher intensity resulted in a larger improvement in endothelial function, indicating that more clinical trials should be conducted to better define the best training intensity for different outcomes [48]. Considering the inverse relationship between exercise volume and intensity, it is reasonable to think that the exercise intensity does not limit additional improvements in glycaemic control but, instead, the lower volume required to perform exercise at this level of effort. In this regard, a 10 week exercise training trial combining moderate cycling with episodes of very intense exercise resulted in important $\mathrm{HbA}_{1 \mathrm{c}}$ reductions in patients with type 2 diabetes [49].

In resistance training programmes, meta-regression models as well as weighted correlations did not show a relationship between intensities and changes in $\mathrm{HbA}_{1 \mathrm{c}}$. Although gains in lean body mass may have a positive association 


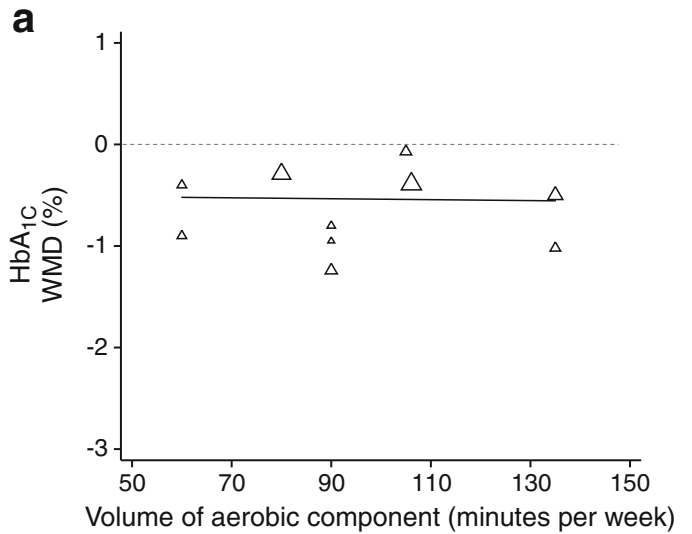

b

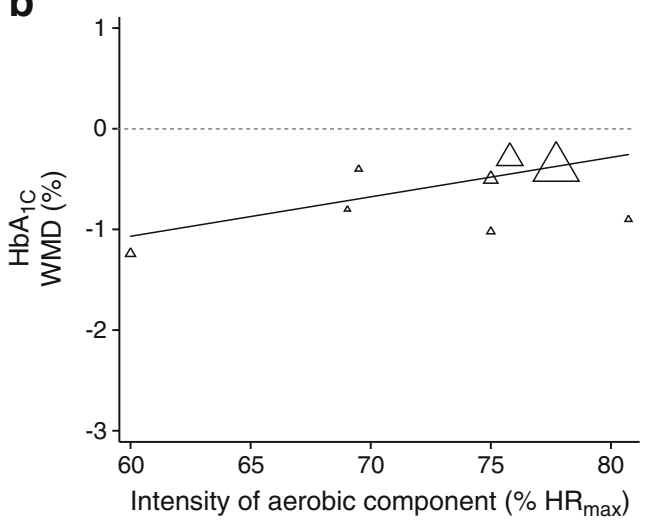

Fig. 4 Association between exercise volume and intensity with $\mathrm{HbA}_{1 \mathrm{c}}$ changes in studies of supervised combined training showing the aerobic and resistance components separately. Scatterplots show both aerobic $(\mathbf{a}, \mathbf{b})$ and resistance $(\mathbf{c}, \mathbf{d})$ components. Exercise volume is expressed as minutes of aerobic exercise per week for the aerobic component or number of sets per week for the resistance component. Likewise, intensity is expressed as percentages of $\mathrm{HR}_{\max }$ or $1-\mathrm{RM}$ for aerobic and resistance components, respectively. Each observation represents the WMD of $\mathrm{HbA}_{1 \mathrm{c}}$ between the combined training group and control group. The size of the triangles is proportional to the

with enhanced insulin action in different populations [50-52], resistance training seems to improve insulin sensitivity and reduce $\mathrm{HbA}_{1 \mathrm{c}}$ even without increases in muscle mass [53], through increases in GLUT4 content and insulin signalling [54]. Therefore, exercise intensity would not necessarily need to induce muscle hypertrophy to improve glucose control. We point out that the exercise frequency was the same (three sessions/week) in all resistance training studies, therefore limiting our analysis to the association between $\mathrm{HbA}_{1 \mathrm{c}}$ and volume of resistance exercise. However, we did not observe significant associations between the number of sets or repetitions with changes in $\mathrm{HbA}_{1 \mathrm{c}}$, suggesting that training volume plays a minor role in glycaemic control for isolated resistance training.

Regarding the combined training studies, the novel finding of our study is that the volume of resistance exercises seems to be the differential characteristic related to glycaemic control,
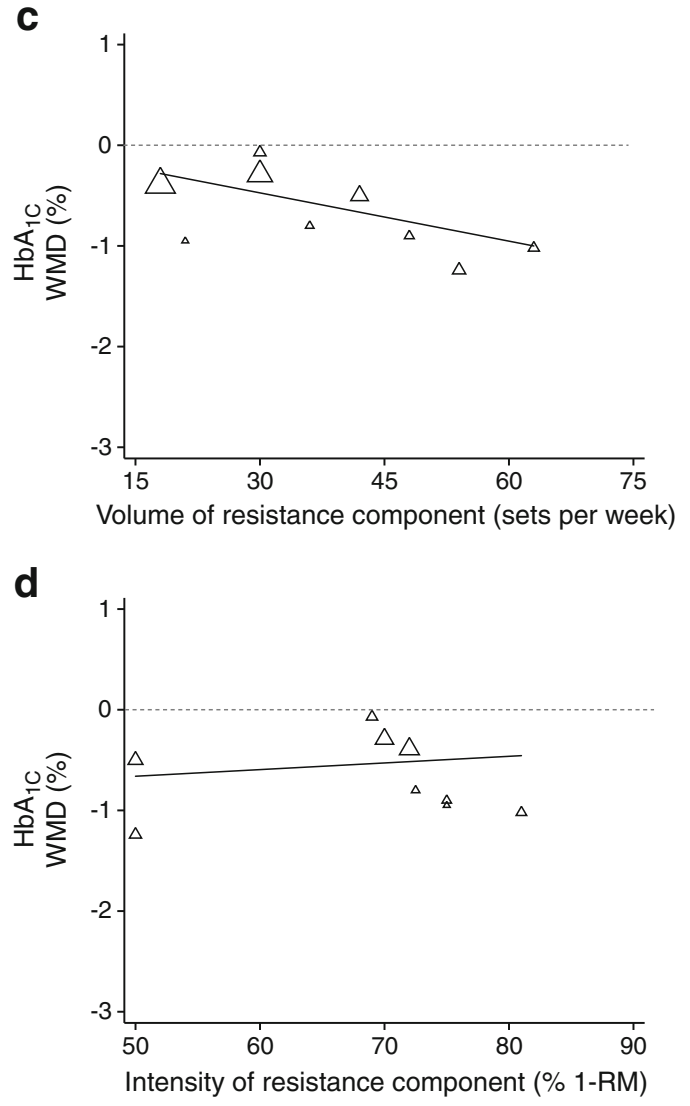

inverse variance of each study in the pooled analysis. Slopes for weighted regressions and weighted correlations were, respectively: $y=10^{-5}-05 x-0.65$ and $r=-0.08, p=0.8$ for the volume of aerobic component, and $y=0.02 x-2.5$ and $r=0.67, p=0.07$ for the intensity of aerobic component. For the resistance components, slopes for weighted regressions and weighted correlations were, respectively: $y=-0.02-0.10$ and $r=-0.70, p=0.04$ for the volume of the resistance component, and $y=0.001 x-0.76$ and $r=0.27, p=0.5$ for the intensity of the resistance component

as reflected by the inverse association between the number of sets per week and $\mathrm{HbA}_{1 \mathrm{c}}$ levels. Given that the mean duration of the aerobic component in combined interventions was 33 min per session, we would expect larger $\mathrm{HbA}_{1 \mathrm{c}}$ reductions when more sets of resistance exercises are added to that amount of aerobic exercise. Supporting this notion, results from an RCT comparing aerobic, resistance and combined training also suggest the importance of exercise volume in lowering $\mathrm{HbA}_{1 \mathrm{c}}$ after combined training programmes [9]. Although our analysis of the distinct aerobic and resistance components indicates that resistance exercise volume most importantly underlies the benefits of combined training, it is still a matter of discussion whether this type of training could induce additional $\mathrm{HbA}_{1 \mathrm{c}}$ reductions when exercise volume is comparable with that of aerobic or resistance training alone $[8$, $9,55,56]$. Considering the results shown, we speculate that there should be a minimal amount of aerobic exercise 
( $\sim 33 \mathrm{~min}$ ) so that the effects of high-volume resistance exercise could be elicited in combined exercise programmes. Moreover, although the findings may seem counterintuitive, the resistance exercise in combined training is not comparable with the high intensity of aerobic exercise. This result suggests that, once the volume is appropriate, the use of additional resistive exercises will also induce larger reductions in $\mathrm{HbA}_{1 \mathrm{c}}$ levels.

One strength of this study is that we performed a systematic review to quantitatively assess weighted estimates for the characteristics related to the effects of supervised exercise training. It indicates reductions of approximately $0.6 \%$ in level of $\mathrm{HbA}_{1 \mathrm{c}}$, which is numerically comparable with the effect of adding non-insulin glucose-lowering drugs to metformin therapy [57]. Further, it extends the knowledge for designing exercise prescriptions aiming to optimise glycaemic control in type 2 diabetes. By assessing several variables in aerobic training studies, we identified the importance of frequency of sessions. Furthermore, we conducted separate analyses of combined training studies and added insight on the likely role of resistance exercise as part of combined aerobic/resistance training. Therefore, the present study demonstrates objective training factors that may be further investigated in RCTs as well as suggesting the need to address differences in exercise training in future guidelines.

As increased exercise intensity might reduce the compliance with supervised exercise programmes [58], our findings underscore the importance of recommending more frequent exercise sessions for patients with type 2 diabetes [1].

There are some limitations to our analysis. First, as a systematic review of published literature, we extracted information that was sometimes dynamic throughout the studies, such as progressive exercise durations and/or intensities. Therefore, our results have a potential bias due to the use of averages generated in secondary data analysis. To minimise this effect, we contacted authors either to clarify issues around the methods used or to obtain additional data. When the information was considerably imprecise (e.g. exercise intensity in some studies), data were not entered in qualitative analyses. Second, we analysed only supervised exercise interventions, which may not be feasible for all patients with type 2 diabetes. Thus, our findings are relevant to centrebased exercise prescriptions, but cannot be generalised to all exercise programmes in type 2 diabetes. We point out that, despite well-known health benefits, physical activity recommendations alone have not been shown to be effective in improving glycaemic control [2, 6, 7], highlighting the need for improved strategies to deliver these interventions. Finally, the present study suggests the need for a large multi-arm trial comparing different regimens of exercise training focusing on either intensity or volume for patients with type 2 diabetes. Reduction in $\mathrm{HbA}_{1 \mathrm{c}}$ is associated with exercise frequency in supervised aerobic training, and with weekly volume of resistance exercise in supervised combined training. Therefore, exercise volume is a major determinant of glycaemic control in patients with type 2 diabetes following structured exercise programmes.

Acknowledgements We are grateful to study authors S. Balducci ("La Sapienza" University, Rome, Italy), R. J. Sigal (University of Calgary, Canada), M. L. Jorge (University of Uberlândia, Brazil), S. Kurban (University of Selcuk, Turkey) and N. P. Kadoglou (University of Athens, Greece) for clarifying details of their study methods or providing additional data from their studies.

D. Umpierre, B. D. Schaan and P. A. B. Ribeiro are greatly thankful to the mentorship of J. P. Ribeiro (in memoriam). He will be sadly missed, but his enduring and substantial legacy will be intensely alive.

Funding This work was partially supported by Conselho Nacional de Desenvolvimento Científico e Tecnológico (CNPq, doctoral scholarship 142209/2009), Coordenação de Aperfeiçoamento de Pessoal de Nível Superior (PNPD CAPES-UFRGS), and FIPE/HCPA.

Duality of interest JPR reported having received lecturing fees from AstraZeneca and Servier, and grant support from Boehringer Ingelheim, Bristol-Myers Squibb, Merck Sharp \& Dohme, Servier and Takeda. The other authors declare no duality of interest associated with this manuscript.

Contribution statement DU, BDS and JPR conceived and designed the project. DU and PABR reviewed the retrieved studies and conducted data extraction. DU conducted data analyses. DU, PABR, BDS and JPR were responsible for data interpretation. DU drafted the manuscript, and BDS and JPR revised it critically for intellectual contributions. BDS and JPR coordinated the study development. All authors reviewed and edited the manuscript. All authors read and approved the final manuscript.

\section{References}

1. American Diabetes Association (2012) Standards of medical care in diabetes. Diabetes Care 35(Suppl 1):S11-S63

2. Umpierre D, Ribeiro PA, Kramer CK et al (2011) Physical activity advice only or structured exercise training and association with HbA1c levels in type 2 diabetes: a systematic review and metaanalysis. JAMA 305:1790-1799

3. Boulé NG, Kenny GP, Haddad E, Wells GA, Sigal RJ (2003) Meta-analysis of the effect of structured exercise training on cardiorespiratory fitness in type 2 diabetes mellitus. Diabetologia 46:1071-1081

4. Snowling NJ, Hopkins WG (2006) Effects of different modes of exercise training on glucose control and risk factors for complications in type 2 diabetic patients: a meta-analysis. Diabetes Care 29:2518-2527

5. Thomas DE, Elliott EJ, Naughton GA (2006) Exercise for type 2 diabetes mellitus. Cochrane Database Syst Rev, issue 3, art. no.: CD002968. doi:10.1002/14651858.CD002968.pub2

6. Balducci S, Zanuso S, Nicolucci A et al (2010) Effect of an intensive exercise intervention strategy on modifiable cardiovascular risk factors in subjects with type 2 diabetes mellitus: a randomized controlled trial: the Italian Diabetes and Exercise Study (IDES). Arch Intern Med 170:1794-1803 
7. Andrews RC, Cooper AR, Montgomery AA et al (2011) Diet or diet plus physical activity versus usual care in patients with newly diagnosed type 2 diabetes: the Early ACTID randomised controlled trial. Lancet 378:129-139

8. Church TS, Blair SN, Cocreham S et al (2010) Effects of aerobic and resistance training on hemoglobin A1c levels in patients with type 2 diabetes: a randomized controlled trial. JAMA 304:22532262

9. Sigal RJ, Kenny GP, Boulé NG et al (2007) Effects of aerobic training, resistance training, or both on glycemic control in type 2 diabetes: a randomized trial. Ann Intern Med 147:357-369

10. Hawley JA, Gibala MJ (2012) What's new since Hippocrates? Preventing type 2 diabetes by physical exercise and diet. Diabetologia 55:535-539

11. Houmard JA, Tanner CJ, Slentz CA, Duscha BD, McCartney JS, Kraus WE (2004) Effect of the volume and intensity of exercise training on insulin sensitivity. J Appl Physiol 96:101-106

12. Nicolucci A, Balducci S, Cardelli P et al (2012) Relationship of exercise volume to improvements of quality of life with supervised exercise training in patients with type 2 diabetes in a randomised controlled trial: the Italian Diabetes and Exercise Study (IDES). Diabetologia 55:579-588

13. Hanestad BR, Albrektsen G (1991) Quality of life, perceived difficulties in adherence to a diabetes regimen, and blood glucose control. Diabet Med 8:759-764

14. Hansen D, Dendale P, Jonkers RA et al (2009) Continuous low- to moderate-intensity exercise training is as effective as moderate- to high-intensity exercise training at lowering blood $\mathrm{HbA}(1 \mathrm{c})$ in obese type 2 diabetes patients. Diabetologia 52:1789-1797

15. Moher D, Liberati A, Tetzlaff J, Altman DG (2009) Preferred reporting items for systematic reviews and meta-analyses: the PRISMA statement. Ann Intern Med 151(264-269):W264

16. Liberati A, Altman DG, Tetzlaff J et al (2009) The PRISMA statement for reporting systematic reviews and metaanalyses of studies that evaluate health care interventions: explanation and elaboration. Ann Intern Med 151:W65-W94

17. Swain DP, Abernathy KS, Smith CS, Lee SJ, Bunn SA (1994) Target heart rates for the development of cardiorespiratory fitness. Med Sci Sports Exerc 26:112-116

18. Brzycki M (1993) Strength testing: predicting a one-rep max from a reps-to-fatigue. J Phys Health Edu Recreat Dance 64:88-90

19. Harbord R, Higgins J (2009) Meta-regression in Stata. In: Sterne JAC, Newton HJ, Cox NJ (eds) Meta-analysis in Stata. Stata Press, College Station

20. Indrayan A (2008) Medical Biostatistics. Chapman \& Hall/CRC, Boca Raton

21. Higgins JPT (2012) Analysing data and undertaking meta-analysis. In: Higgins JPT, Green S (eds) Cochrane handbook for systematic reviews of interventions 5.1.0 [updated March 2011]. In: The Cochrane Library. www.cochrane-handbook.org. Accessed 20 January 2012

22. Bjorgaas M, Vik JT, Saeterhaug A et al (2005) Relationship between pedometer-registered activity, aerobic capacity and selfreported activity and fitness in patients with type 2 diabetes. Diabetes Obes Metabol 7:737-744

23. Cuff DJ, Meneilly GS, Martin A, Ignaszewski A, Tildesley HD, Frohlich JJ (2003) Effective exercise modality to reduce insulin resistance in women with type 2 diabetes. Diabetes Care 26:29772982

24. Giannopoulou I, Fernhall B, Carhart R et al (2005) Effects of diet and/or exercise on the adipocytokine and inflammatory cytokine levels of postmenopausal women with type 2 diabetes. Metabolism 54:866-875

25. Goldhaber-Fiebert JD, Goldhaber-Fiebert SN, Tristan ML, Nathan DM (2003) Randomized controlled community-based nutrition and exercise intervention improves glycemia and cardiovascular risk factors in type 2 diabetic patients in rural Costa Rica. Diabetes Care 26:24-29

26. Jorge ML, de Oliveira VN, Resende NM et al (2011) The effects of aerobic, resistance, and combined exercise on metabolic control, inflammatory markers, adipocytokines, and muscle insulin signaling in patients with type 2 diabetes mellitus. Metabolism 60:12441252

27. Kadoglou NP, Iliadis F, Angelopoulou N et al (2007) The antiinflammatory effects of exercise training in patients with type 2 diabetes mellitus. Eur J Cardiovasc Prev Rehabil 14:837-843

28. Kadoglou NP, Perrea D, Iliadis F, Angelopoulou N, Liapis C, Alevizos M (2007) Exercise reduces resistin and inflammatory cytokines in patients with type 2 diabetes. Diabetes Care 30:719721

29. Kadoglou NP, Iliadis F, Sailer N et al (2010) Exercise training ameliorates the effects of rosiglitazone on traditional and novel cardiovascular risk factors in patients with type 2 diabetes mellitus. Metabolism 59:599-607

30. Kadoglou NP, Vrabas IS, Kapelouzou A et al (2012) The impact of aerobic exercise training on novel adipokines, apelin and ghrelin, in patients with type 2 diabetes. Med Sci Monit 18:CR290-CR295

31. Kurban S, Mehmetoglu I, Yerlikaya HF, Gönen S, Erdem S (2011) Effect of chronic regular exercise on serum ischemia-modified albumin levels and oxidative stress in type 2 diabetes mellitus. Endocr Res 36:116-123

32. Lambers S, van Laethem C, van Acker K, Calders P (2008) Influence of combined exercise training on indices of obesity, diabetes and cardiovascular risk in type 2 diabetes patients. Clin Rehabil 22:483-492

33. Middlebrooke AR, Elston LM, Macleod KM et al (2006) Six months of aerobic exercise does not improve microvascular function in type 2 diabetes mellitus. Diabetologia 49:2263-2271

34. Raz I, Hauser E, Bursztyn M (1994) Moderate exercise improves glucose metabolism in uncontrolled elderly patients with noninsulin-dependent diabetes mellitus. Isr J Med Sci 30:766-770

35. Ribeiro IC, Iborra RT, Neves MQ et al (2008) HDL atheroprotection by aerobic exercise training in type 2 diabetes mellitus. Med Sci Sports Exerc 40:779-786

36. Sridhar B, Haleagrahara N, Bhat R, Kulur AB, Avabratha S, Adhikary P (2010) Increase in the heart rate variability with deep breathing in diabetic patients after 12-month exercise training. Tohoku J Exp Med 220:107-113

37. Vancea DM, Vancea JN, Pires MI, Reis MA, Moura RB, Dib SA (2009) Effect of frequency of physical exercise on glycemic control and body composition in type 2 diabetic patients. Arq Bras Cardiol 92:23-30

38. Verity LS, Ismail AH (1989) Effects of exercise on cardiovascular disease risk in women with NIDDM. Diabetes Res Clin Pract 6:27-35

39. Castaneda C, Layne JE, Munoz-Orians L et al (2002) A randomized controlled trial of resistance exercise training to improve glycemic control in older adults with type 2 diabetes. Diabetes Care 25:2335-2341

40. Dunstan DW, Daly RM, Owen N et al (2002) High-intensity resistance training improves glycemic control in older patients with type 2 diabetes. Diabetes Care 25:1729-1736

41. Balducci S, Leonetti F, Di Mario U, Fallucca F (2004) Is a longterm aerobic plus resistance training program feasible for and effective on metabolic profiles in type 2 diabetic patients? Diabetes Care 27:841-842

42. Dobrosielski DA, Gibbs BB, Ouyang P et al (2012) Effect of exercise on blood pressure in type 2 diabetes: a randomized controlled trial. J Gen Intern Med 27:1453-1459

43. Loimaala A, Huikuri HV, Koobi T, Rinne M, Nenonen A, Vuori I (2003) Exercise training improves baroreflex sensitivity in type 2 diabetes. Diabetes 52:1837-1842 
44. Tessier D, Menard J, Fulop T et al (2000) Effects of aerobic physical exercise in the elderly with type 2 diabetes mellitus. Arch Gerontol Geriatr 31:121-132

45. Kang J, Robertson RJ, Hagberg JM et al (1996) Effect of exercise intensity on glucose and insulin metabolism in obese individuals and obese NIDDM patients. Diabetes Care 19:341-349

46. Oshida Y, Yamanouchi K, Hayamizu S, Sato Y (1989) Long-term mild jogging increases insulin action despite no influence on body mass index or $\mathrm{VO}_{2}$ max. J Appl Physiol 66:2206-2210

47. Seals DR, Hagberg JM, Hurley BF, Ehsani AA, Holloszy JO (1984) Effects of endurance training on glucose tolerance and plasma lipid levels in older men and women. JAMA 252:645-649

48. da Silva CA, Ribeiro JP, Canto JC et al (2012) High-intensity aerobic training improves endothelium-dependent vasodilation in patients with metabolic syndrome and type 2 diabetes mellitus. Diabetes Res Clin Pract 95:237-245

49. Mourier A, Gautier JF, de Kerviler E et al (1997) Mobilization of visceral adipose tissue related to the improvement in insulin sensitivity in response to physical training in NIDDM. Effects of branched-chain amino acid supplements. Diabetes Care 20:385391

50. Miller JP, Pratley RE, Goldberg AP et al (1994) Strength training increases insulin action in healthy 50- to 65-yr-old men. J Appl Physiol 77:1122-1127

51. Poehlman ET, Dvorak RV, DeNino WF, Brochu M, Ades PA (2000) Effects of resistance training and endurance training on insulin sensitivity in nonobese, young women: a controlled randomized trial. J Clin Endocrinol Metab 85:2463-2468

52. Ryan AS, Pratley RE, Goldberg AP, Elahi D (1996) Resistive training increases insulin action in postmenopausal women. $\mathrm{J}$ Gerontol A Biol Sci Med Sci 51:M199-M205

53. Misra A, Alappan NK, Vikram NK et al (2008) Effect of supervised progressive resistance-exercise training protocol on insulin sensitivity, glycemia, lipids, and body composition in Asian Indians with type 2 diabetes. Diabetes Care 31:1282-1287

54. Tabata I, Suzuki Y, Fukunaga T, Yokozeki T, Akima H, Funato K (1999) Resistance training affects GLUT-4 content in skeletal muscle of humans after 19 days of head-down bed rest. J Appl Physiol 86:909-914

55. Armstrong MJ, Boulé NG, Sigal RJ (2011) Exercise interventions and glycemic control in patients with type 2 diabetes. JAMA 306:607

56. Ribeiro JP, Schaan BD, Umpierre D (2011) Exercise interventions and glycemic control in patients with type 2 diabetes. JAMA 306:608-609

57. Gross JL, Kramer CK, Leitão CB et al (2011) Effect of antihyperglycemic agents added to metformin and a sulfonylurea on glycemic control and weight gain in type 2 diabetes: a network metaanalysis. Ann Intern Med 154:672-679

58. King AC, Haskell WL, Young DR, Oka RK, Stefanick ML (1995) Long-term effects of varying intensities and formats of physical activity on participation rates, fitness, and lipoproteins in men and women aged 50 to 65 years. Circulation 91:2596-2604 